\title{
Voice Outcomes After Laryngeal Microsurgery With Adjunctive Steroid Injection for Reinke Edema
}

\author{
Jeon Yeob Jang (i) - Dong Young Kim (iD $\cdot$ Ga Young Lee (i) $\cdot$ So Hyoung Park (i) $\cdot$ Hyeran Lee (i) \\ Yoo Seob Shin (D) Chul-Ho Kim (i) \\ Department of Otolaryngology, Ajou University Hospital, Ajou University School of Medicine, Suwon, Korea
}

Reinke edema (RE) is characterized by diffuse polypoid degeneration of the superficial layer of the lamina propria, and it commonly presents with edematous or polypoid vocal folds [1]. Conservative management, such as smoking cessation and voice therapy, is initially recommended; however, it is frequently ineffective [2]. Various surgical procedures have been reported to lead to partial improvements in voice quality, although the role of surgery remains under discussion [3,4]. Interestingly, recent studies have proposed that intralesional steroid injections may improve phonatory outcomes and provide an alternative treatment option for patients with RE [5,6]. In this study, we aimed to evaluate the clinical outcomes after the concomitant use of laryngeal microsurgery and a steroid injection for the treatment of RE.

This study included 40 consecutive patients with RE who underwent laryngeal microsurgery with an adjunctive steroid injection between January 2014 and September 2018 at Ajou University Hospital. The study was approved by the Institutional Review Board of Ajou University Hospital, and the requirement for written informed consent was waived due to the retrospective nature of the study.

Surgical interventions were performed under general anesthesia. Surgical exposure was obtained using a suspension laryngoscope and operating microscope. A longitudinal mucosal incision was made on the superior surface of the vocal fold using a $\mathrm{CO}_{2}$ laser ( $1 \mathrm{~W}$, superpulse, continuous). A mucosal microflap was made, and mucoid gelatinous fluid in the Reinke space was meticulously evacuated using forceps or suction. Excessive mucosa

\footnotetext{
- Received August 14, 2020

Revised November 10, 2020

Accepted November 20, 2020

- Corresponding author: Chul-Ho Kim

Department of Otolaryngology, Ajou University School of Medicine and

Department of Molecular Science and Technology, Ajou University,

164 World cup-ro, Yeongtong-gu, Suwon 16499, Korea

Tel: +82-31-219-5269, Fax: +82-31-219-5264

E-mail: ostium@ajou.ac.kr
}

of the vocal fold was excised using cold instruments, and the remaining epithelium was repositioned as a microflap over the lamina propria. Bleeding control was maintained using a cottonoid soaked in 1:10,000 epinephrine saline solution. During the surgical procedure, 0.1 to $0.3 \mathrm{~mL}$ of dexamethasone sodium phosphate (5 mg/mL; Yuhan Inc., Seoul, Korea) was injected with a $25-\mathrm{G}$ injection needle into the midportion of the lamina propria of the vocal folds to avoid a deeper injection into the vocalis muscle. If the lesion was bilateral, steroid injections were administered into both vocal folds.

Each patient underwent a voice evaluation and video laryngoscopy before surgery, 2 weeks after surgery, and 3 months after surgery. Maximal phonation time (MPT) was measured using the Phonatory Aerodynamic System Model 6600 (KayPentax, Lincoln Park, NJ, USA). Acoustic analysis was performed using the Multidimensional Voice Program software package (KayPentax). Perceptual voice evaluations were performed by speechlanguage pathologists. The overall grade and roughness ratings were used for analyses ( $0=$ normal, $1=$ slight disturbance, $2=$ moderate disturbance, and $3=$ severe disturbance).

SPSS ver. 18.0 (SPSS Inc., Chicago, IL, USA) was used to conduct the statistical analysis. Values are presented as the mean \pm standard deviation. Significant differences between two groups were determined using the Wilcoxon signed-rank test. To compare multiple groups, the Friedman test or the Wilcoxon signed-rank test with the Bonferroni correction was used to determine statistical significance. The $P$-values $<0.05$ were considered to indicate statistical significance.

The mean age of the subjects was 53.43 years. Most of the patients were current smokers, and $40 \%$ had a history of voice abuse (Supplementary Table 1). The clinical grade of RE was obtained through the analysis of video laryngoscopy images, as previously reported [7]. There were 13 patients with mild (clinical grades 1-2) lesions and 27 patients with severe (clinical grades 3-4) lesions (Supplementary Fig. 1A). The mean preoperative clinical grade was 2.85. Evaluations of postoperative video

Copyright @ 2021 by Korean Society of Otorhinolaryngology-Head and Neck Surgery.

This is an open-access article distributed under the terms of the Creative Commons Attribution Non-Commercial License (https://creativecommons.org/licenses/by-nc/4.0) which permits unrestricted non-commercial use, distribution, and reproduction in any medium, provided the original work is properly cited. 
laryngoscopy images showed significantly improved grades after surgery (Fig. 1, Supplementary Fig. 1B).

Next, the degree of voice improvement was analyzed (Table 1). Although aerodynamic analyses showed that the MPT did not significantly increase, the acoustic analyses of jitter, shimmer, and noise-to-harmonic ratio (NHR) showed statistically significant improvements after surgery. In addition to the objective voice analysis, subjective evaluations using the grade, roughness, breathiness, asthenia, and strain (GRBAS) scale also showed significant improvements in overall grade and roughness. To evaluate changes in pitch, the fundamental frequency was measured
(Fig. 1). The mean fundamental frequency was $129.05 \mathrm{~Hz}$ overall, $119.18 \mathrm{~Hz}$ in men, and $151.27 \mathrm{~Hz}$ in women preoperatively. Statistically significant increases in the overall fundamental frequency were found after surgery. In men, the improvements in fundamental frequency were not statistically significant (119.18 $\mathrm{Hz}$ preoperatively, $137.69 \mathrm{~Hz}$ at 2 weeks after surgery, 136.09 $\mathrm{Hz}$ at 3 months after surgery; $P=0.085)$. In women, the improvements in fundamental frequency were statistically significant $(151.57 \mathrm{~Hz}$ preoperatively, $197.31 \mathrm{~Hz}$ at 2 weeks after surgery, $188.53 \mathrm{~Hz}$ at 3 months after surgery; $P=0.018$ ). However, a further analysis of the other voice parameters, including MPT,
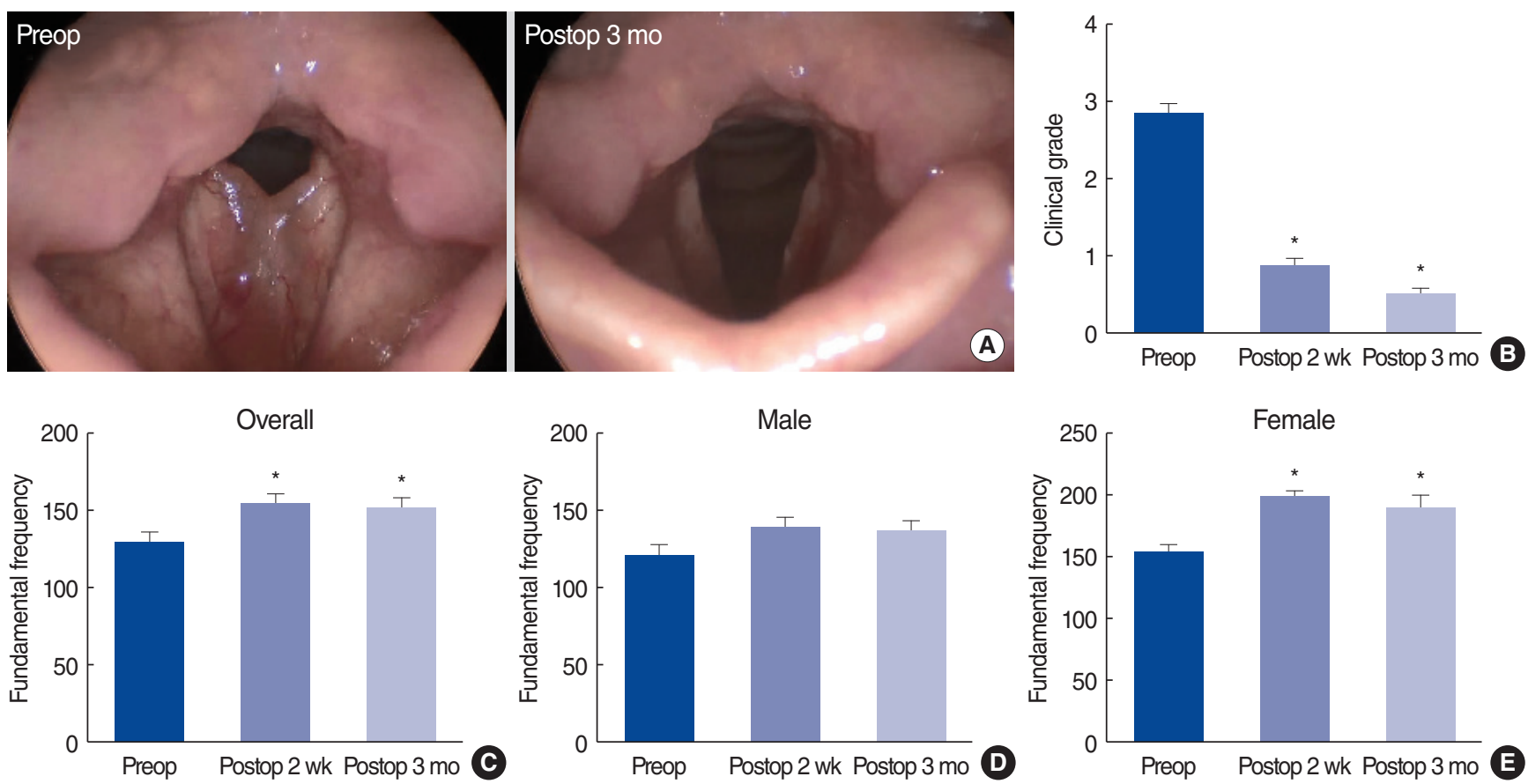

Fig. 1. Comparisons of clinical grade and fundamental frequency before and after surgery. (A, B) Videolaryngoscopic findings and quantifications of clinical grade before and after surgery. (C-E) Comparisons of fundamental frequency. Preop, preoperative; Postop, postoperative. ${ }^{\star} P<0.05$ vs. preoperative status.

Table 1. Comparisons of preoperative and postoperative voice characteristics in patients with Reinke edema

\begin{tabular}{|c|c|c|c|c|c|c|}
\hline Phonatory result & Preoperative & Postoperative 2 wk & Postoperative $3 \mathrm{mo}$ & $P$-value ${ }^{a)}$ & $P$-value ${ }^{b)}$ & $P$-value ${ }^{c)}$ \\
\hline \multicolumn{7}{|l|}{ Aerodynamic } \\
\hline MPT (sec) & $11.33 \pm 5.27$ & $13.22 \pm 5.42$ & $16.58 \pm 9.59$ & 0.108 & 0.060 & 0.018 \\
\hline \multicolumn{7}{|l|}{ Acoustic } \\
\hline Jitter (\%) & $3.29 \pm 2.39$ & $1.87 \pm 1.86$ & $1.90 \pm 2.43$ & 0.007 & 0.012 & 0.016 \\
\hline Shimmer (\%) & $10.26 \pm 5.22$ & $6.38 \pm 4.98$ & $6.40 \pm 3.92$ & $<0.001$ & $<0.001$ & $<0.001$ \\
\hline $\mathrm{NHR}$ & $0.26 \pm 0.18$ & $0.17 \pm 0.10$ & $0.17 \pm 0.12$ & $<0.001$ & 0.006 & $<0.001$ \\
\hline \multicolumn{7}{|l|}{ GRBAS scale } \\
\hline Overall grade & $2.38 \pm 0.68$ & $1.27 \pm 0.80$ & $1.20 \pm 0.83$ & $<0.001$ & $<0.001$ & $<0.001$ \\
\hline Roughness & $1.89 \pm 0.99$ & $0.49 \pm 0.87$ & $0.66 \pm 0.92$ & $<0.001$ & $<0.001$ & $<0.001$ \\
\hline
\end{tabular}

Values are presented as mean \pm standard deviation.

MPT, maximal phonation time; NHR, noise-to-harmonic ratio; GRBAS, grade, roughness, breathiness, asthenia, and strain.

a)Comparisons among the three groups, Friedman test. ${ }^{b}$ Comparisons between preoperative and postoperative (2 weeks) characteristics, Wilcoxon signed-rank test with the Bonferroni correction. ${ }^{\mathrm{c}}$ Comparisons between preoperative and postoperative ( 3 months) characteristics, Wilcoxon signed-rank test with the Bonferroni correction. 
jitter, shimmer, and NHR, showed more prominent improvements in men than in women (Supplementary Table 2). Although it is difficult to draw firm conclusions due to the small number of subjects, the possibility of differential treatment outcomes according to sex may be further investigated in the future with multi-institutional studies.

The degree of voice improvement was evaluated separately in each subgroup of patients based on the clinical grade of RE (Supplementary Table 3). In patients with grades 1-2, MPT and jitter did not improve significantly, while other parameters, including shimmer, NHR, and GRBAS score, did show improvements. In patients with grades $3-4$, all of the analyzed voice parameters showed statistically significant improvements after surgery. Intralesional steroid injections have emerged as an alternative treatment for benign vocal fold lesions, including RE [8]. Based on the hypothesis that benign vocal fold lesions are related to abnormal inflammatory processes, local steroid injections are expected to disrupt the pathophysiology of these lesions and have been reported to show promising results in selected patients [9]. However, the recurrence rate is relatively high, indicating the limited efficacy of intralesional steroid injections when used alone for RE [6].

Adjunctive steroid injections after laryngeal microsurgery have recently been reported to be safe and associated with improved voice quality in patients with benign vocal fold lesions [10]. In that study, adjunctive steroid injections were associated with a 0.3 -fold lower risk of persistent dysphonia after surgery; however, patients with RE were not enrolled as the study subjects [10]. Since excessive fibrosis and scarring of the vocal folds after surgery may hamper mucosal vibration, the use of steroid injections is reasonable to reduce fibrosis of the vocal folds.

One potential issue associated with steroid injections is delayed wound healing, as steroids have anti-inflammatory effects. However, no surgery-related complications were observed in the present study. Video laryngoscopy revealed no significant surgical defects on the vocal folds. Thus, injection of $0.1-0.3 \mathrm{~mL}$ of dexamethasone during RE surgery might be safe and feasible. In conclusion, our results suggest that conservative surgery with an adjunctive steroid injection is feasible and might be considered as a useful treatment option to restore voice quality and pitch in patients with RE. However, this was a single-arm study without a comparative group, which limits the conclusions. Future prospective randomized trials are needed to confirm the efficacy of adjunctive steroid injections.

\section{CONFLICT OF INTEREST}

No potential conflict of interest relevant to this article was reported.

\section{ORCID}

Jeon Yeob Jang https://orcid.org/0000-0002-9047-4896

Dong Young Kim https://orcid.org/0000-0001-9368-9705

Ga Young Lee https://orcid.org/0000-0001-6051-6648

So Hyoung Park https://orcid.org/0000-0003-1355-139X

Hyeran Lee https://orcid.org/0000-0003-0722-419X

Yoo Seob Shin https://orcid.org/0000-0002-9855-3185

Chul-Ho Kim https://orcid.org/0000-0003-4250-6504

\section{AUTHOR CONTRIBUTIONS}

Conceptualization: JYJ, YSS, CHK. Data curation: DYK, GYL, SHP, HL. Formal analysis, Methodology, Project administration, Visualization, \& Writing-original draft: JYJ. Writing-review \& editing: CHK.

\section{SUPPLEMENTARY MATERIALS}

Supplementary materials can be found via https://doi.org/10. 21053/ceo.2020.01746.

\section{REFERENCES}

1. Marcotullio D, Magliulo G, Pezone T. Reinke's edema and risk factors: clinical and histopathologic aspects. Am J Otolaryngol. 2002 Mar-Apr;23(2):81-4.

2. Hojslet PE, Moesgaard-Nielsen V, Karlsmose M. Smoking cessation in chronic Reinke's oedema. J Laryngol Otol. 1990 Aug;104(8): 626-8.

3. Khodeir MS, Hassan SM, El Shoubary AM, Saad MN. Surgical and nonsurgical lines of treatment of reinke's edema: a systematic literature review. J Voice. 2019 Nov 21 [Epub]. https://doi.org/10.1016/ j.jvoice.2019.10.016.

5. Tateya I. Laryngeal steroid injection. Curr Opin Otolaryngol Head Neck Surg. 2009 Dec;17(6):424-6.

6. Lee SW, Park KN. Long-term efficacy of percutaneous steroid injection for treating benign vocal fold lesions: a prospective study. Laryngoscope. 2016 Oct;126(10):2315-9.

7. Tan M, Bryson PC, Pitts C, Woo P, Benninger MS. Clinical grading of Reinke's edema. Laryngoscope. 2017 Oct;127(10):2310-3.

8. Wang CT, Liao LJ, Cheng PW, Lo WC, Lai MS. Intralesional steroid injection for benign vocal fold disorders: a systematic review and meta-analysis. Laryngoscope. 2013 Jan;123(1):197-203.

9. Rafii B, Sridharan S, Taliercio S, Govil N, Paul B, Garabedian MJ, et al. Glucocorticoids in laryngology: a review. Laryngoscope. 2014 Jul; 124(7):1668-73.

10. Cho JH, Kim SY, Joo YH, Park YH, Hwang WS, Sun DI. Efficacy and safety of adjunctive steroid injection after microsurgical removal of benign vocal fold lesions. JVoice. 2017 Sep;31(5):615-20. 